\title{
Significados otorgados a la evaluación de la enseñanza y el aprendizaje. Interpretación a partir de un grupo de maestros de educación física
}

\author{
Meanings Given to the Assessment of Teaching and Learning. Interpretation \\ from a Group of Physical Education Teachers
}

\author{
Significados outorgados à avaliação do ensino e da aprendizagem. Interpretação \\ de um grupo de professores de educação física
}

\section{Beatriz Chaverra Fernández. ${ }^{a}$}

a Instituto Universitario de Educación Física y Deporte, Universidad de Antioquia. Fono: 2199252. Correo electrónico: biatachf@yahoo.es

\begin{abstract}
RESUMEN
Este texto presenta algunos resultados de la investigación realizada sobre los significados que le otorgan los maestros a la evaluación en educación física. El diseño metodológico es cualitativo con un enfoque hermenéutico. Los participantes fueron cinco maestros de educación física de enseñanza primaria con diversidad de años de experiencia docente. Se utilizó la entrevista en profundidad como técnica principal para la recolección de la información. Los resultados determinan que los significados otorgados por los maestros a la evaluación, están orientados hacia la reflexión y mejora del proceso enseñanza-aprendizaje. Sus ideas y creencias, están más vinculadas hacia una racionalidad práctica. La evaluación de los aprendizajes es asumida de manera participativa y permanente, buscando desarrollar actitudes de autonomía y reflexión en los estudiantes. Sin embargo, la evaluación de la enseñanza es un camino poco explorado, no se encuentran acciones sistemáticas y planificadas a este respecto.

Palabras clave: educación física, pensamiento del profesor, evaluación, enseñanza.
\end{abstract}

\begin{abstract}
This paper presents some results of a research on the meanings given by teachers of physical education to the assessment. The methodology used is qualitative with a hermeneutic approach. The participants of this study were five primary school physical teachers with different years of teaching experience, and in-depth interviews were the main technique for data collection. The results suggest that the meaning given by teachers to the evaluation, are oriented toward reflection and improvement of the teaching-learning process. Their ideas and beliefs are mainly linked to practical rationality. The evaluation of learning is conceived as permanent and participatory, seeking to develop attitudes of autonomy and reflection upon students. However, the evaluation of teaching is an unexplored path, and there are no systematic or planned actions in this regard.
\end{abstract}

Key words: physical education, teacher's thinking, assessment, teaching.

\section{RESUMO}

Apresentam-se alguns resultados da pesquisa sobre os significados que os professores dão à avaliação em Educação Física. A metodologia foi a abordagem hermenêutica qualitativa. Os participantes foram cinco professores de Educação Física com diversos anos de experiência no ensino primário. Foram usadas entrevistas em profundidade como a principal técnica de coleta de dados. Resultados sugerem que o significado dado à avaliação pelos professores é orientado pela reflexão e melhoria do processo de ensino-aprendizagem. Suas ideias e crenças estão mais ligadas à racionalidade prática. A avaliação da aprendizagem é assumida de forma permanente e participativa, buscando desenvolver nos estudantes atitudes de autonomia e de reflexão. Embora a avaliação do ensino seja um caminho inexplorado, não existem ações sistemáticas e planejadas a este respeito.

Palavras chave: educação física, pensamento do professor, avaliação, ensino. 
Estudios Pedagógicos XL, $\mathrm{N}^{\circ}$ 2: 65-82, 2014

SIGNIFICADOS OTORGADOS A LA EVALUACIÓN DE LA ENSEÑANZA Y EL APRENDIZAJE. INTERPRETACIÓN A PARTIR DE UN GRUPO DE MAESTROS DE EDUCACIÓN FÍSICA

\section{INTRODUCCIÓN}

Sin lugar a dudas, la evaluación es uno de los aspectos educativos que ha generado mayores dificultades en el proceso enseñanza-aprendizaje (López-Pastor et al., 2013), algunos autores han llegado a considerarla como el elemento más complejo de la educación (Cabezas, González y Carpintero, 2009; Salinas, 2002; Santos, 2003). Esta concepción se basa en los múltiples factores que determinan las ideas y estilos de evaluación, por ejemplo, el concepto que tenga el docente de conocimiento, enseñanza, educación, aprendizaje, estudiante, etc., guiará directamente su manera de evaluar (Brown, 2002), de ahí la importancia de profundizar en sus esquemas de pensamiento.

La evaluación forma parte de estructuras conceptuales más amplias, no sólo está relacionada con el paradigma de educación física en que se apoya, sino también con la concepción de currículo e idea de educación que se tiene en un momento histórico determinado. López (1999) vincula la evaluación en educación física a la idea de racionalidad no sólo asumida como sinónimo de conocimiento, sino también, en términos de Habermas, "con la forma en que los sujetos capaces de lenguaje y de acción hacen uso del conocimiento" (1987: 24), en este sentido, propone la racionalidad técnica y la racionalidad práctica como un conjunto de pensamientos y prácticas que permiten identificar las formas de actuar del profesorado ante la evaluación (López, 1999).

La racionalidad técnica está amparada en las ciencias naturales y especialmente en el positivismo, donde la medición y la "objetividad" son los principios más importantes para conocer los fenómenos. Este tipo de pensamiento científico pretende dar respuesta a los problemas humanos, incluidos los problemas de la educación, aplicando un modelo técnico centrado en el control y la productividad.

Esta característica conduce a que la evaluación constituya un fin en sí misma y gire en torno a ella el proceso de enseñanza, ya que si todo lo enseñado es demostrado por el estudiante en la evaluación, será una prueba "objetiva" de que fue un buen proceso de enseñanza. En consecuencia, el papel que desempeña el estudiante es pasivo y no tiene más participación que la otorgada por la respuesta ante un cuestionamiento o la ejecución ante una prueba. En esta misma línea, el papel del maestro no es otro que ser un calificador y verificador de los aprendizajes, por tanto, la heteroevaluación es la manera más común en que se relacionan los actores.

Esta racionalidad técnica ha marcado fuertemente la manera de evaluar en educación física. La finalidad ha estado dominada por la medición del cuerpo, con el objetivo fundamental de comparar los resultados con unos parámetros estandarizados.

Esta forma de evaluación está íntimamente ligada a los objetivos operativos que propone una visión de educación física tradicional anclada a elementos cuantificables y verificables del movimiento. Para identificar estos aprendizajes se ha acudido a instrumentos fiables y técnicas cuantitativas, entre las más destacadas encontramos las baterías de test de condición física y de habilidad motriz (Cit. en Penney et al., 2009: 345) o Physical Fitness Tests (López, 2006). Estos test fueron incluidos en el contexto educativo como imitación de las evaluaciones biológicas, para dar cuenta de los aprendizajes y el desarrollo de habilidades motrices de los estudiantes. Como lo plantea López (2000), la mayoría de los test que se utilizan en Educación física, provienen del rendimiento motor y la antropometría, campos de conocimiento cercanos a las ciencias biomédicas.

Como alternativa a este paradigma tradicional surge la racionalidad práctica, amparada en una concepción cualitativa de la educación y más cercana a los paradigmas interpreta- 
tivos, comprensivos y fenomenológicos. Este nuevo enfoque de la evaluación ha tomado diversos nombres en la literatura: evaluación alternativa, evaluación auténtica, evaluación para el aprendizaje o evaluación integral (López et al., 2013), aunque el término más común es evaluación formativa.

Desde esta racionalidad, la evaluación se convierte en parte fundamental del sistema educativo, ya que pretende mejorar el proceso enseñanza-aprendizaje y a la vez convertirse en herramienta investigativa. Para lograrlo, rescata el papel activo de los actores implicados en el proceso y la convierte en una oportunidad de diálogo y construcción colectiva.

Esta perspectiva más integral asume la evaluación como un proceso ético, que invita al docente a pensarse como un sujeto reflexivo y concebir otras formas de evaluar los aprendizajes, rescatando al estudiante como un interlocutor válido, capaz de opinar y emitir juicios sobre su propio aprendizaje y sobre la misma enseñanza.

Una característica importante de esta racionalidad es que reivindica la evaluación de la enseñanza como una posibilidad para el aprendizaje. Así, el docente debe reflexionar sobre la calidad de sus procesos empleados, la pertinencia de sus acciones pedagógicas en conformidad con los objetivos de formación, todo con el fin de tomar decisiones que mejoren el aprendizaje y su enseñanza.

Desde hace algunos años se vienen desarrollando investigaciones y reflexiones conceptuales alrededor de la evaluación en educación física desde una concepción formativa y alternativa al modelo tradicional (Desrosiers, Genet-Volet \& Godbout, 1997; Díaz, 2005; López, 2004, 2000, 1999; MacPhail \& Halbert, 2010; Ní Chóinín y Cosgrave, 2012; Pérez, Heras y Herrán, 2008; Velázquez y Hernández, 2004). El cambio paradigmático que se propone en la evaluación, está dado por estos avances teóricos y con ellos, una invitación a los docentes a modificar sus ideas y creencias, para que este nuevo paradigma se haga realidad en el día a día de las aulas.

Surge en este punto un elemento determinante: el pensamiento del profesor. En el ámbito internacional se ha consolidado una línea de investigación que explora las relaciones que existen entre lo que piensan los docentes y lo que hacen en el aula (Clark y Peterson, 1990; Marcelo, 1987; Shulman, 1989). Así, las concepciones epistemológicas, metodológicas e ideológicas vienen revisándose intentando dar cuenta de los vínculos que puedan establecerse entre el pensamiento y el proceso enseñanza-aprendizaje.

La enseñanza en Educación Física como proceso interpersonal e intencionado, no sólo incluye el aprendizaje de habilidades y destrezas motrices específicas, sino también el desarrollo de capacidades humanas fundamentales para el desarrollo humano y social de los estudiantes (Uribe, Gallo y Castro, 2004), por tanto, "moviliza las capacidades, los pensamientos, y las acciones de profesores y alumnos" (Carreiro da Costa, 2004: 56). Por esta razón, indagar sobre los procesos de pensamiento de sus actores se convierte en objeto de estudio obligado para mejorar el proceso enseñanza-aprendizaje y la educación física como área.

García (2003) presenta la evolución de la investigación sobre el pensamiento del profesor partiendo de los estudios más cercanos al paradigma proceso-producto, pasando a la evolución del estudio del pensamiento y conocimiento del profesor desde los enfoques cognitivos (la toma de decisiones preactivas e interactivas), hasta los enfoques alternativos (conocimiento práctico y conocimiento didáctico del contenido). No obstante, Carreiro da Costa $(2008,2004)$ define que el análisis de los procesos de pensamiento del profesor de educación física ha incidido sobre cuatro grandes áreas: a) las creencias, valores, percepciones y preocupaciones; b) la planificación y evaluación; c) la relación entre 
la planificación y el comportamiento de profesor y alumnos, y; d) los conocimientos y problemas prácticos.

Son diversos los autores que han realizado investigaciones en cada una de estas líneas (algunos son: Ayvazo \& Ward, 2011; Azevedo, 2009; Correa y Martínez, 2006; Díaz, 2001; Ennis \& Zhu, 1991; Fernández, 2007; Henrique, 2004; Januario, 1992; Jinhong, 2012; Pradas, 2010; Tristán, 2010) con diversidad de población, contextos y objetivos, sin embargo, al indagar más detenidamente en la relación pensamiento del profesor-evaluación, se encuentra que ha sido un tema poco abordado, sólo los estudios realizados por Guío (2012), Vera (2001) y Vera y Moreno (2007) hacen referencia a esta relación, destacando la fuerte conexión entre pensamiento y acción evaluativa que aún está por descubrirse.

Según lo anterior, el vínculo entre el pensamiento del profesor y la evaluación en educación física es un objeto de estudio escasamente explorado. ¿Cómo planifican la evaluación? ¿Para qué evalúan? ¿Qué papel cumple la evaluación en su proceso de enseñanza? ¿Qué implicaciones tiene la evaluación que realiza en el proceso formativo de los estudiantes? ¿Cómo es la participación de los estudiantes en el proceso evaluativo? ¿Qué influencia tiene la formación inicial en las prácticas evaluativas que realiza?, es decir, surgen diversas preguntas sobre las prácticas evaluativas que aún están por ser abordadas.

Como un aporte a estos interrogantes, esta investigación indagó sobre el significado que le otorgan los maestros a la evaluación con el interés de conocer desde sus propios relatos cómo inciden en ellos las prácticas evaluativas que realizan, qué reflexiones surgen a partir del proceso o cómo los transforman, dicho de otro modo, se buscó identificar el sentido que le confieren a la evaluación como un elemento cotidiano en sus aulas. La pregunta que orientó esta investigación fue: ¿cuál es el sentido que le otorgan los maestros de educación física a la evaluación de la enseñanza y el aprendizaje?

\section{METODOLOGÍA}

Este estudio se desarrolló con un diseño metodológico de corte cualitativo con un enfoque hermenéutico, el cual se enmarca en el paradigma interpretativo comprensivo. Con el término investigación cualitativa suele entenderse el tipo de investigación que por sus características se puede utilizar para "obtener detalles complejos de algunos fenómenos, tales como sentimientos, procesos de pensamiento y emociones, difíciles de extraer o de aprehender por métodos de investigación más convencionales" (Strauss y Corbin, 2002: 13). Tales procesos de pensamiento fueron el centro de atención de este estudio, identificando los significados que le otorgan los maestros a la evaluación y por ende a sus prácticas evaluativas.

La hermenéutica, entendida como ciencia y arte de la interpretación de los discursos, fue la estrategia metodológica determinante para el proceso de análisis de la información. Como lo apunta Habermas, "La investigación hermenéutica de la realidad sólo es posible bajo el interés determinante de conservar y ampliar la intersubjetividad en la comprensión orientadora de posibles acciones" (1982: 69).

Esta alternativa de investigación cualitativa, aparece como una opción que no se agota exclusivamente en su dimensión filosófica, sino que trasciende a una propuesta metodológica, donde la comprensión de la realidad se asume bajo la metáfora de un texto, el cual es susceptible de interpretaciones (Sandoval, 1996). Metodológicamente, la hermenéutica requiere comprender los textos a partir de un ejercicio interpretativo 
intencional, donde se traspasen las fronteras de la palabra literal para comprender el sentido con el cual se expresan.

Para esta investigación, los textos fueron las entrevistas realizadas a los maestros, tanto su expresión oral, como su transcripción al papel. Asimismo, fueron elementos de interpretación los documentos aportados por los participantes.

\subsection{PARTICIPANTES}

Los participantes de este estudio fueron cinco maestros de educación física que actualmente imparten el área en la enseñanza primaria en las comunidades autónomas de Madrid y Castilla-La Mancha (España).

El muestreo de la investigación fue intencional, lo que Goetz y LeCompte (1988) llaman selección basada en criterios. Todos los maestros participantes son hombres, con diversidad de años de experiencia y formación. Como parte de la confidencialidad de los datos, sus nombres han sido cambiados, en la Tabla 1 se presenta una breve caracterización de los participantes en relación a la edad, titulación, años de experiencia profesional, y ciclo de enseñanza primaria que orientaba al momento del estudio.

Tabla 1. Caracterización de los participantes de la investigación

\begin{tabular}{|c|c|c|c|c|}
\hline Nombre & $\begin{array}{c}\text { Edad } \\
\text { (años) }\end{array}$ & Titulación & $\begin{array}{c}\text { Años de } \\
\text { experiencia } \\
\text { profesional }\end{array}$ & $\begin{array}{c}\text { Ciclo de } \\
\text { enseñanza } \\
\text { actual }\end{array}$ \\
\hline Héctor & 28 & $\begin{array}{c}\text { - Maestro especialidad } \\
\text { Educación Física. } \\
\text { - Licenciado en Ciencias del } \\
\text { Deporte y la Actividad Física. }\end{array}$ & $\begin{array}{c}5 \text { años y } 7 \\
\text { meses }\end{array}$ & Tercero \\
\hline Juan & 29 & $\begin{array}{c}\text { - Maestro especialidad } \\
\text { Educación Física. } \\
\text { - Licenciado en Ciencias del } \\
\text { Deporte y la Actividad Física. }\end{array}$ & $\begin{array}{c}3 \text { años y } 6 \\
\text { meses }\end{array}$ & $\begin{array}{c}\text { Segundo y } \\
\text { tercero }\end{array}$ \\
\hline Antonio & 29 & $\begin{array}{c}\text { - Maestro especialidad } \\
\text { Educación Física. } \\
\text { - Licenciado en Ciencias del } \\
\text { Deporte y la Actividad Física. }\end{array}$ & 5 años & Tercero \\
\hline Pablo & 24 & $\begin{array}{l}\text { - Maestro especialidad } \\
\text { Educación Física. }\end{array}$ & $\begin{array}{l}\text { - Maestro especialidad en } \\
\text { Educación Física. }\end{array}$ & $\begin{array}{c}\text { Primero y } \\
\text { segundo }\end{array}$ \\
\hline
\end{tabular}

Fuente: elaboración propia. 


\subsection{TÉCNICAS E INSTRUMENTOS}

La entrevista es la más común y una de las más importantes herramientas de recolección de información en la investigación cualitativa (Denzin \& Lincoln, 2005; Myers \& Newman, 2007), en este estudio se optó por emplear la entrevista en profundidad.

Este tipo de entrevista requiere encuentros entre el investigador y los participantes para comprender la visión que tienen sobre el tema de investigación expresado en sus propias palabras (Taylor y Bogdan, 1987). Los encuentros siguieron un modelo de conversación discursiva que permitió conocer las experiencias y concepciones de los maestros sobre la evaluación, y a partir de allí, interpretar su significado y sentido. Si bien la entrevista en profundidad es una conversación oral, se elaboró un guion de preguntas, que pasó por la validación de un grupo de profesores(as) universitarios expertos(as) en educación física y evaluación para determinar su validez.

Otra técnica utilizada fue el análisis documental, el cual permitió complementar, contrastar y validar la información obtenida en la entrevista. En este caso los documentos de carácter oficial como los denomina Woods (1987) fueron proporcionados por los maestros para su análisis (instrumentos, unidades didácticas, programaciones).

También se utilizó como instrumento el diario del entrevistador (Taylor y Bogdan, 1987) donde fue registrada diversa información sobre lo acontecido en las entrevistas: una descripción general de lo ocurrido, interpretaciones, intuiciones, expresiones no verbales de los maestros; que luego dieron luces para analizar la información.

\subsection{ANÁLISIS DE LA INFORMACIÓN}

Para analizar la información tuvimos en cuenta la propuesta de Taylor y Bogdan (1987) quienes plantean que debe ser un proceso en continuo progreso durante la investigación, e iniciar el análisis profundo de la información recolectada lo más pronto posible, realizándose éste de forma paralela a la recolección de información. Estos autores proponen tres fases para el análisis de los datos. La primera es la fase de descubrimiento, en la cual se identificaron categorías y temas, desarrollando además conceptos y proposiciones. Para realizar este paso, se hizo una lectura detallada de todas las entrevistas y los documentos aportados por los maestros. En esta lectura, se fueron registrando ideas y reflexiones que emergían de los datos en función del objetivo de investigación.

En la fase de codificación se refinaron las interpretaciones de los datos y la comprensión del objeto de estudio. En esta fase se realizó un listado preliminar de categorías, se codificaron los datos y se redefinió el análisis de toda la información. La categorización se realizó por vía inductiva, es decir, a partir de las ideas y el conocimiento previo de las teorías, se hizo lectura de los discursos, esperando que de ellos emergieran las categorías. Una vez identificadas las principales categorías de la codificación, se repasó la lista inicial nuevamente y se realizó una depuración. Los datos se codificaron de acuerdo al tipo de información suministrada y el nombre de los maestros, por ejemplo: E. H. o E. L., lo cual significará, al leer los resultados, que se trató de la Entrevista de Héctor o Luis.

En la fase de relativización de los datos se buscó interpretar la información a la luz del contexto en que acontecieron. En esta fase se tuvo en cuenta no generalizar lo dicho por los maestros, sino comprenderlo desde la realidad que cada uno vive y revisarlo en virtud de los elementos teóricos sobre el tema y las investigaciones actuales. 
Las entrevistas fueron trascritas conservando el lenguaje y la expresión de los participantes. Las categorías y subcategorías siguen la propuesta de Strauss y Corbin (2002) desde la codificación axial. Los supuestos surgen de la experiencia propia como docente y del diálogo con los maestros participantes de la investigación, también de la relación con los elementos teóricos sobre el tema y las orientaciones que sobre evaluación tiene el currículo español.

La triangulación se desarrolló por medio del contraste teórico. Los datos sumistrados por los participantes fueron contrastados con las investigaciones actuales y teorías sobre el tema abordado. Este contraste teórico también se presentó entre el discurso y los documentos entregados por los participantes. Como criterio de credibilidad se hizo una devolución del texto (transcripciones) a los participantes para su revisión.

Los entrevistados firmaron un consentimiento informado, el cual explicaba los objetivos de la investigación, la técnica de recolección de información, los compromisos voluntarios que adquirían al participar del estudio y la utilización de la información recolectada.

\section{RESULTADOS Y DISCUSIÓN}

\subsection{PENSAMIENTOS Y CREENCIAS DE LOS MAESTROS RESPECTO A LA EVALUACIÓN}

En los discursos de los maestros no se encuentran conceptos o significados que hagan alusión a la evaluación como elemento de medición y control, por el contrario, se percibe en sus palabras una concepción de evaluación al servicio de los actores implicados en el proceso enseñanza-aprendizaje con miras a mejorarlo. Se podría decir que esta visión está en la línea de los resultados del estudio de Guío, quien concluye que la evaluación es asumida por los profesores desde un sentido humanista y fundamentalmente cualitativa donde se reconoce al estudiante en todas sus dimensiones "y sus propósitos son esencialmente educativos" (2012: 867).

Para los participantes de este estudio la evaluación como un proceso permite la reflexión, el análisis y el mejoramiento de su labor docente. Por ende, se puede decir que tienen una concepción de evaluación más inclinada hacia una racionalidad práctica.

E. A.: Evaluar no es una prueba específica concreta... me acerco a la manera de evaluar, de reflexionar sobre lo acontecido.

En esta línea, las finalidades que le otorgan los maestros a la evaluación, es decir, el para qué realizan sus procesos evaluativos, se pueden vincular a las dos concepciones que plantea López (1999): como proceso de reflexión y como proceso de análisis y mejora.

La evaluación como un proceso de reflexión implica reconocerse como un profesional que debe pensar su labor docente, es decir, asumirse como un profesional reflexivo (Schön, 1992), que requiere una visión abierta hacia las situaciones y actuaciones que realiza día a día en las aulas. Para López, una característica de la evaluación como reflexión es el pensar sobre "los éxitos y los fracasos (dentro de los aprendizajes) y la necesidad de una evaluación y reflexión sobre los propios errores como requisito de cambio" (1999: 130). Al respecto, un maestro dice:

E. H.: El proceso de evaluación lo que implica es mucho de cabeza, mucho pensamiento, mucho reflexionar y, más que evaluación, es reflexionar cómo solucionar los problemas que 
te encuentras, es ir modificando no la evaluación, sino la enseñanza, el proceso de evaluación, es modificar la enseñanza para que el alumno consiga esto, el alumno consiga lo otro.

Este ejercicio de pensar sobre su labor como maestros, permite tomar conciencia sobre las acciones que se llevan a cabo con los estudiantes, buscar estrategias de cambio en beneficio del proceso enseñanza-aprendizaje, y asumirse como sujetos críticos con su labor.

Por su parte, la finalidad de la evaluación referida al proceso de análisis y mejora, se puede identificar en las ideas de los maestros que se refieren a la evaluación como medio que permite verificar la enseñanza y el aprendizaje o realizar un cambio si fuera necesario.

E. J.: Antes te decía que la evaluación debe ir a la reflexión, y a saber, y a pensar por qué no salen bien las cosas, o por qué a este niño le pasa esto, o por qué este niño no ha hecho las cosas.

Esta concepción de la evaluación como mejora está centrada prioritariamente sobre el aprendizaje. Reconocer la evaluación como una oportunidad para mejorar la enseñanza es una postura muy tímida que aparece en frases aisladas. Se podría pensar que si bien hay un esfuerzo por comprender la evaluación desde una concepción formativa, todavía hay una tendencia muy fuerte a dirigir la evaluación sólo hacia los estudiantes.

\subsubsection{Las transformaciones en evaluación}

Esta visión integral y formativa de la evaluación no acompaña a los maestros desde sus inicios en la docencia. Todos reconocen que son los años de experiencia profesional los que les han permitido reflexionar y realizar acciones que antes no hubieran imaginado.

Sus conceptos y prácticas iniciales en la docencia y especialmente en relación a la evaluación, estaban ligadas a los aprendizajes que habían adquirido en la Universidad y principalmente al procedimiento selectivo que realiza España para ingresar al Cuerpo de Maestros (Oposición). Sin embargo, el inicio de la vida laboral permite confrontar los aprendizajes anteriores con las realidades a las que se enfrentan en las aulas de clase. La teoría deja de ser el único motor de las acciones, para reconocer en la experiencia una fuente de aprendizaje mayor.

Este acercamiento a la vida laboral genera un impacto con la realidad que lleva al maestro a reflexionar y reestructurar sus pensamientos alrededor de la evaluación, y especialmente sus prácticas. Luego de varios años de experiencia, reconocen que sus prácticas evaluativas no eran tan formativas como son ahora; la intencionalidad, el momento y los procedimientos e instrumentos han cambiado.

E. H.: Al empezar estaba más preocupado del control, entonces la evaluación llegaba al final y decía: bueno este lo ha hecho bien...no utilizaba los instrumentos. Luego ya cuando tenía más claras las unidades, ya ibas generándote una pequeña ficha para cada unidad y lo haces al final; luego ya vas avanzando y metes fichas entre ellos o algún trabajillo... entonces la evaluación es cuestión de años, es cuestión de experiencia.

Se corroboran los resultados de la investigación realizada por Hein, Vieira y Mendes (2007) en que los profesores consideran la experiencia profesional como el elemento que 
más influye en la restructuración de las prácticas evaluativas. Estos cambios no se presentan de manera esporádica, ni por la acumulación de años en las aulas, es necesario que haya un interés formativo en los maestros que los lleven a cuestionar sus pensamientos y acciones, sustentadas en la reflexión sobre su cotidianidad. Es así como el conocimiento pedagógico de los maestros se transforma y consolida a partir de la experiencia docente.

Las concepciones, creencias y pensamientos que tienen los docentes sobre la evaluación, influyen directamente sobre sus prácticas, y a la vez, estas prácticas reconfiguran sus pensamientos y creencias, se manifiesta como una relación dialógica que permite una constante revisión de su labor.

\subsection{EVALUACIÓN DEL APRENDIZAJE EN EDUCACIÓN FÍSICA. UN PROCESO QUE REQUIERE COMPROMISO}

Identificar el sentido que los maestros dan a la evaluación del aprendizaje comporta varios elementos que confluyen en sus prácticas. Los procedimientos, instrumentos y momentos relacionados con sus prácticas evaluativas, varían según sus experiencias y motivaciones.

Desligar procedimientos, instrumentos, momentos y actores de la evaluación no resulta tarea fácil cuando analizamos un discurso, estos elementos se entremezclan para dar forma al estilo y concepción de evaluación de un maestro. Sin embargo, intentaremos subdividir estos elementos para una mejor comprensión de los sentidos que le otorgan los maestros a la evaluación del aprendizaje y cómo la llevan a cabo.

\subsubsection{Instrumentos y actores de la evaluación}

En educación física reconocemos que los diferentes test que miden el cuerpo y las capacidades físicas, han sido los instrumentos dominantes y casi exclusivos para evaluar en el área. Los maestros de esta investigación no escapan a esta tradición y reconocen que hubo momentos donde llegaron a hacer uso de ellos, pero reflexionaron sobre su finalidad formativa y ahora no los conciben en sus prácticas.

E. P.: Yo al principio hacia esos test en tercer ciclo [11-12 años] y empecé a valorar que tampoco veía mucho sentido, porque los niños mejoran en velocidad, resistencia, fuerza por el propio crecimiento físico, más que por lo que tú haces en clase, y porque en primaria no debe haber trabajo específico de eso, entonces dejé de hacerlo.

Los instrumentos que emplee un maestro para evaluar, no dan cuenta por sí solos de las finalidades que persiguen con ellos, será sólo cuando utilice los resultados obtenidos, que se pueda interpretar la intencionalidad formativa o no de aquellos procesos. Por esta razón, el test por sí mismo no debe eliminarse, tal vez, los maestros no han encontrado el sentido formativo que pueden tener y son descartados por su tradición anclada en la racionalidad técnica y por no encontrar otra manera de abordarlos desde una concepción más formativa.

Otros instrumentos utilizados para evaluar los aprendizajes están ligados a procedimientos más cualitativos y, en la medida de las posibilidades, a la sistematización cotidiana de los procesos por medio de la observación principalmente.

Se puede decir que la heteroevaluación que realiza el maestro hacia el estudiante, está basada en procedimientos de observación y descripción, acompañados de instrumentos 
como planillas, diarios de campo o fichas estructuradas. En educación física, la observación se convierte en un proceso fundamental para identificar las falencias o avances de los estudiantes, según Díaz (2005), en ocasiones será suficiente con observar a los estudiantes mientras ellos realizan la tarea.

A través de las observaciones, los maestros llevan un registro de los acontecimientos que suceden en las clases, lo que les permite recordar la situación del grupo en determinado momento y los hechos más relevantes en relación a los aprendizajes. Parece ser que observar se convierte en la mejor técnica para identificar los avances o dificultades que se presentan en el proceso de enseñanza-aprendizaje.

En los discursos de los maestros no sólo se evidencia la heteroevaluación, sino que manifiestan la importancia de la participación activa de los estudiantes en todo el proceso evaluativo, por tanto, realizan actividades coevaluativas y autoevaluativas por medio de instrumentos más o menos estructurados.

Para la coevaluación se proponen actividades en parejas o grupos, acompañadas de instrumentos estructurados que no generen dudas a los estudiantes. Un ejemplo de instrumento para realizar coevaluación y autoevaluación es el aportado por Héctor. Esta ficha la usa en una unidad didáctica de Kinball con los estudiantes de tercer ciclo (12 años).

Tabla 2. Instrumento de coevaluación y autoevaluación de una unidad didáctica de Kinball aportado por un profesor

\begin{tabular}{|c|c|c|c|c|c|c|}
\hline \multirow{2}{*}{\multicolumn{2}{|c|}{$\begin{array}{l}\text { ALUMNO: } \\
\text { * En la primera columna evalúate a ti mismo, y en el resto a los } \\
\text { compañeros de tu grupo. } \\
\text { * Rellena la tabla con los siguientes indicadores: } \\
S \text { (Siempre) }-\boldsymbol{N}(\text { Nunca })-\boldsymbol{A V} \text { (A veces) }\end{array}$}} & \multicolumn{5}{|c|}{ CURSO: } \\
\hline & & \multirow[t]{2}{*}{$\dot{-}$} & \multirow[t]{2}{*}{ i } & \multirow[t]{2}{*}{$\dot{m}$} & \multirow[t]{2}{*}{$\dot{r}^{\dagger}$} & \multirow[t]{2}{*}{$\dot{\varphi}$} \\
\hline 1. & Forma correctamente el trípode de ataque & & & & & \\
\hline 2. & Nombra adecuadamente el ataque: "OMNIKIN..." & & & & & \\
\hline 3. & Golpea el balón con los dos brazos al mismo tiempo & & & & & \\
\hline 4. & Acepta decisiones de los compañeros & & & & & \\
\hline 5. & Respeta las decisiones del árbitro & & & & & \\
\hline
\end{tabular}

Fuente: participante de la investigación.

En relación a la autoevaluación, los maestros destacan la importancia de crear en los niños y niñas hábitos de reflexión desde pequeños, como un aporte a su educación, aunque todos los maestros admiten que es un proceso complejo debido a su edad. El estudio realizado por Ureña et al. (2006) demuestra que la edad y el grado de madurez de los estudiantes son grandes inconvenientes al momento de realizar un proceso de autoevaluación. Sin embargo, puede subsanarse la dificultad cuando los instrumentos se adaptan a las posibilidades de los estudiantes. 
Los maestros de esta investigación piensan en posibles soluciones para esta dificultad, e intentan involucrar a los estudiantes más pequeños en un proceso de evaluación formativa a través de cuadernos o diarios, hojas de registro y fichas estructuradas.

E. J.: Por ejemplo con caritas, con dibujos o simplemente con unas palabras, o sea, en función del nivel que tengan.

La coevaluación y la autoevaluación se hacen presentes en los discursos de los maestros y se corroboran en los instrumentos aportados. Estas prácticas las realizan con la intencionalidad de brindar participación a los estudiantes en su proceso de aprendizaje y, a la vez, fomentar en ellos actitudes de autonomía y responsabilidad.

Es de resaltar que los instrumentos presentados por los maestros son construcciones propias que surgen a partir de sus experiencias y necesidades, como es el caso de los deportes alternativos de Kinball y Botebol. Asimismo, estos instrumentos han cambiado según el contexto en que se desenvuelven y las características de los niños y niñas.

\subsubsection{Momentos de la evaluación}

La recolección de la información a través de los instrumentos y por los actores relacionados, se presenta en determinados momentos del proceso enseñanza-aprendizaje. En los discursos de los maestros se evidencia la importancia de realizar una evaluación inicial, sin embargo, hay una indeterminación en relación a la evaluación procesual y final.

La evaluación inicial o diagnóstica la sustentan en la necesidad de obtener información sobre un elemento determinado para iniciar el proceso de enseñanza-aprendizaje.

E. H.: Lo que suelo hacer en clase de educación física es una evaluación inicial, lo inicial para conocer... siempre suelo hacer una evaluación inicial aunque sea un diagrama con ellos en clase, ponerles un esquema que tengo yo de juegos, “a ver ¿Quéjuegos habéis jugado?”. Éstos.

Tal como se evidencia en los instrumentos, la observación se constituye en el procedimiento privilegiado entre los maestros. Esta acción de observar y escribir en sus diarios o fichas la realizan en varios momentos, algunos intentan hacerlo diariamente, aunque reconocen la dificultad que esto conlleva por el poco tiempo que disponen para hacerlo, sin embargo, buscan estrategias o momentos para hacer pequeñas anotaciones, que luego pueden ampliar o, simplemente, les da pistas sobre lo que ocurrió en la clase.

E. J.: Es complicado, siempre lo suelo hacer si no tengo reunión, o entre clase y clase hacer unas pequeñas anotaciones que ya luego yo voy enlazando en el diario, y bueno, no siempre se ocupa la extensión que me gustaría, pero bueno es lo que intento.

Si bien se refleja una intencionalidad de registrar algunas acciones que realizan día a día, no se evidencia claramente cómo llevan a cabo la evaluación durante las unidades didácticas; sus explicaciones se entremezclan entre la evaluación inicial, formativa y final.

E. H.: No es algo que hagas al principio, que hagas al final, que hagas en medio porque sí, sino que hay una conexión entre toda la evaluación que llevas hasta el final de curso. 
Estudios Pedagógicos XL, $\mathrm{N}^{\circ}$ 2: 65-82, 2014

SIGNIFICADOS OTORGADOS A LA EVALUACIÓN DE LA ENSEÑANZA Y EL APRENDIZAJE. INTERPRETACIÓN A PARTIR DE UN GRUPO DE MAESTROS DE EDUCACIÓN FÍSICA

E. J.: Pues sobre todo una evaluación, digamos, continua y global, o sea, que ellos sepan de donde parten, que sepan su nivel, tanto de conocimientos como su nivel de habilidad, como el interés y la motivación que tienen hacia el aprendizaje.

Aunque no son muy claros en su descripción, los momentos en que realizan las actividades evaluativas no se alejan de lo que han manifestado anteriormente en relación a su idea de evaluación como proceso, y no como un momento específico para cuantificar.

\subsection{EVALUACIÓN DE LA ENSEÑANZA. UN PROCESO AÚN POR DESCUBRIR}

Asumir la evaluación como un proceso de regulación y apoyo para mejorar la docencia, implica asumir que la enseñanza y las acciones que realiza el maestro deben ser igualmente evaluadas y reflexionadas.

Para los maestros de este estudio, no revierte igual implicación y dedicación la evaluación de la enseñanza como la evaluación del aprendizaje. Existe una gran inclinación a hacer partícipes a los estudiantes de su evaluación, sin embargo, esta situación no es igual al evaluar la enseñanza. Los maestros reconocen la importancia de realizar este proceso de evaluación a su quehacer docente, pero no se evidencian procesos sistemáticos o concretos para realizarlos. La regularidad entre los maestros entrevistados fue evaluar su enseñanza a partir de conversaciones informales con sus compañeros o autoevaluaciones ante determinadas situaciones.

La autoevaluación se presenta como un ejercicio informal, que es llevado a cabo a partir de las situaciones cotidianas que se presentan en las instituciones educativas. En sus discursos se perciben algunos destellos de reflexión y cuestionamiento hacia su labor, intentando mejorar su actuación con los estudiantes.

E. H.: Tú estás enseñando a los alumnos y se te ocurre algo raro que enseñarles y te funciona, es otra cosa que te va mejorando, y piensas, "pues esto no lo hacía del todo bien", es autoevaluación que te permite mejorar.

E. A.: Yo tengo algún archivo por ahí en el ordenador: "de cara al año que viene”, cosas que he debido mejorar.

La autoevaluación se puede constituir en un proceso de autoformación y crecimiento profesional a partir de las reflexiones en el acto de la clase, o en las conversaciones con los compañeros, pero este proceso requiere estar siempre dispuestos a la auto-observación y análisis de sus prácticas.

Se percibe en los maestros una intencionalidad por pensarse y reflexionar su enseñanza, pero no manifiestan un proceso sistemático o permanente para llevarlo a cabo en cualquiera de las fases de la enseñanza (pre-activa, interactiva y post-activa) (Jackson, 1992), por el contrario, son reflexiones aisladas, determinadas por situaciones específicas y momentáneas.

Esta situación se repite con la coevaluación. Los maestros acuden a la conversación con otros compañeros para compartir sus ideas, intereses, dudas o preguntas sobre su acción como maestros. Estos encuentros están enmarcados en un ejercicio evaluativo realizado de manera informal que no precisa de instrumentos o procedimientos estructurados.

E. H.: Nosotros en tutorías sí que nos evaluamos con otro profesor, no de forma institucionalizada, pero sí de forma en la que tú preguntas, oye ¿Qué te parece esto? y te 
van contestando, "pues bueno, creo que te has equivocado con este alumno cuando le has dicho no sé qué...”.

E. L.: Si como nos juntamos y a veces... oye qué tal, qué estáis haciendo y cómo los ves y tal, así informal.

Una de las razones por la cual no se lleva a cabo un proceso más riguroso en la coevaluación puede ser por la falta de espacios para ello en las instituciones educativas. La cotidianidad de las instituciones no permite tener momentos para que los maestros realicen un proceso conjunto de evaluación de la enseñanza.

Por su parte, no son sólo las dinámicas institucionales sino, en definitiva, la tradición que tenemos los maestros al respecto. La ausencia de evaluación sobre la enseñanza no es sorpresiva si reconocemos que una de las patologías de la evaluación (Santos, 1995) es sólo evaluar al estudiante, además, es relativamente reciente el impulso que la evaluación formativa y la investigación sobre la enseñanza han proporcionado para observar al docente y al sistema educativo en general como objetos de evaluación (Álvarez, 2001; Gimeno, 1992; Hernández y López, 2004) lo que implica tiempo para afianzar estas premisas.

Y si la autoevaluación y la coevaluación aparecen de manera tímida, la heteroevaluación es un camino poco explorado. Realizar un proceso de heteroevaluación de los estudiantes hacia los maestros era un ejercicio casi impensable hace algunos años, sin embargo, las nuevas formas de comprender la educación y especialmente la evaluación, reconocen la importancia de este proceso y les devuelven la voz a los estudiantes para que participen con un juicio crítico en esa evaluación.

En el caso de los maestros participantes, ellos reconocen la evaluación como un mecanismo de reflexión y ajuste hacia el proceso enseñanza-aprendizaje a partir de los aprendizajes de los estudiantes, sin embargo, la evaluación de su enseñanza no es un asunto muy desarrollado. En teoría, todos saben que debe hacerse, pero en la práctica es una cuestión compleja.

E. P.: El profesor también tiene que evaluar su práctica ¿no?, no sólo debe evaluar al alumno, entonces en teoría eso se debe hacer, luego está el paso dos, si eso se hace en la práctica, entonces eso está quedando un poco a nivel individual.

La complejidad puede estar dada por el desconocimiento o por la edad de los estudiantes. En este último caso, los maestros manifiestan que realizar una heteroevaluación con niños(as) es muy complejo. Se refieren a la edad como el principal problema para llevarla a cabo, tal y como se mencionó en la evaluación de los aprendizajes.

E. A.: Desde tu labor como profesor es muy difícil que te hagan una crítica. No tienen un juicio, son pequeños.

Un maestro intentó realizar un ejercicio de heteroevaluación con los estudiantes, tal como le habían enseñado en su Universidad que debía hacerse, sin embargo, el resultado no fue positivo, por ello, ha desistido de esta actividad.

E. A.: Yo por ejemplo lo probé en el primer año, ¡como hay que evaluar!, y es absurdo en primaria, o la manera de utilizarlo ha sido absurda. ¿Qué te parece el profesor? Todo maravilloso... y no lo volví a hacer. 
Tal vez la inexperiencia, la motivación, la falta de tiempo o el desconocimiento de cómo realizar este proceso, pueden ser la razones para que los maestros no realicen la heteroevaluación de la enseñanza. A pesar de esta situación, realizan algunas acciones a este respecto como preguntarles a los estudiantes por situaciones particulares.

E. L.: ¿Qué les ha gustado más?, es lo que les pregunto yo a los niños más pequeños ¿Qué os ha gustado menos y porqué? Y ya según lo que te digan puedes pensar, bueno pues para la siguiente clase, en vez de hacer esto con estas pelotas; voy a hacerlo con otras más grandes, por ponerte un ejemplo, según lo que me hayan dicho ellos.

Tal vez uno de los mayores retos de los maestros es buscar formas de involucrar a los estudiantes en la evaluación de la enseñanza, creer que los(as) niños(as), a pesar de su edad, puedan aportar algún comentario que favorezca su reflexión o permita mejorar lo que hacen diariamente en las clases. Si bien los estudiantes no harán reflexiones profundas sobre elementos metodológicos o teóricos, se deben reconocer como interlocutores válidos, que pueden brindar información sobre sentimientos, acciones y deseos.

Es, en definitiva, el proceso más desatendido por parte de los maestros. En sus discursos y documentos aportados no hay una referencia directa a la evaluación que pueden hacer los estudiantes hacia su enseñanza.

\section{CONCLUSIONES}

La evaluación es un proceso complejo, en el cual intervienen múltiples factores que determinan su desarrollo: la edad de los estudiantes, los contenidos, las características del contexto, los lineamientos legales y curriculares y especialmente la formación y experiencia del maestro. Todos estos elementos intervienen en los pensamientos y prácticas que un docente pueda tener sobre la evaluación.

A partir de los resultados presentados en este estudio, se puede concluir que los significados otorgados por los maestros a la evaluación están relacionados con la reflexión y mejora del proceso enseñanza-aprendizaje. Sus ideas y creencias están más vinculadas a una racionalidad práctica que permite reconocer la evaluación como un ejercicio crítico que revisa las interacciones que se van produciendo entre estudiante, profesor y conocimiento.

En sus discursos, se pueden identificar elementos que evidencian cómo a partir de sus prácticas evaluativas, los pensamientos y prácticas han sido transformados o modificados hacia una visión de evaluación que centra su atención en el mejoramiento del proceso enseñanza-aprendizaje. Las concepciones, creencias y pensamientos que tienen los docentes sobre la evaluación influyen directamente sobre sus prácticas, y a la vez, estas prácticas reconfiguran sus pensamientos y creencias; es una relación dialógica que permite una constante revisión de su labor.

Desde esta perspectiva, la evaluación del aprendizaje es asumida como una acción participativa que pone al estudiante en el centro del proceso. Los maestros manifiestan un gran esfuerzo por evaluar los aprendizajes de manera permanente, construyen instrumentos acordes a la edad, reconocen la importancia de la participación de los estudiantes y, por tanto, utilizan la coevaluación y la autoevaluación. En general, buscan desarrollar actitudes de autonomía y reflexión por parte de los niños y niñas desde edades tempranas. 
Para realizar la evaluación del aprendizaje, la observación se convierte en el procedimiento privilegiado, esta acción, acompañada de fichas o diarios, permite reconocer los logros y dificultades que se presentan en el proceso educativo.

Los instrumentos aportados por los maestros son adecuados al ciclo de enseñanza y, por tanto, a la edad de los estudiantes. Las fichas presentadas para realizar ejercicios de coevaluación y autoevaluación se muestran bien estructuradas, lo que facilita un mejor desarrollo y acción por parte de niños y niñas para lograr los propósitos de enseñanza.

En relación a los contenidos a evaluar, se evidencia en los discursos de los maestros un gran interés por evaluar las actitudes, aunque en los instrumentos sí se hacen evidentes los procedimientos y conceptos. Esta situación puede deberse a la necesidad de privilegiar las actitudes como un medio de formación integral y no sobrevalorar los elementos técnicos del área. De igual manera, los maestros referencian la importancia de valorar los esfuerzos y el comportamiento en relación a la edad en que están los niños y niñas de primaria, y no sólo los elementos técnicos.

En cuanto a la evaluación de la enseñanza, no se encuentran acciones sistemáticas y planificadas hacia la evaluación de su quehacer, y se presenta como un camino poco explorado. Las razones son variables, sin embargo, las más relevantes se relacionan con el tiempo que disponen para hacerlo y de encontrar el camino más apropiado para que sus estudiantes en todos los grados puedan participar y aportar.

Los estudiantes no tienen voz en este proceso, puede ser por su edad o por no encontrar la manera de vincularlos, pero aún así, los estudiantes están desdibujados cuando se habla de evaluar la labor docente.

En relación a la evaluación de la enseñanza, las acciones que realizan quedan relegadas a conversaciones informales con los compañeros de la institución educativa. Se realizan autoevaluaciones sobre situaciones concretas que suceden en sus aulas, pero no se evidencia un proceso de recolección de información, análisis y toma de decisiones durante un periodo de tiempo determinado. No hay sistematicidad en este ejercicio, no se evidencian elementos que permitan asumir que hay una evaluación en las fases preactivas y activas; las pocas alusiones realizadas por los maestros se centran en las fases post-activas.

La ausencia de la evaluación de la enseñanza puede deberse a varios aspectos: las directrices del sistema educativo, las dinámicas institucionales, la formación del maestro, sus experiencias, intereses o motivaciones hacia el proceso evaluativo, etc., es decir, no se puede juzgar por qué este proceso no se lleva a cabo, sólo proponer que los maestros piensen en las razones que los llevan a actuar de determinadas formas y no de otras.

La evaluación, desde una racionalidad práctica, es un paradigma que va ganando terreno en nuestros centros educativos, y especialmente en los pensamientos de los maestros, por tanto, no se puede esperar que sea una cuestión del todo o nada, sino reconocer los grandes avances que puede hacer un maestro desde su realidad y contexto.

\section{REFERENCIAS BIBLIOGRÁFICAS}

Álvarez, J. (2001). Evaluar para conocer, examinar para excluir. Madrid: Morata.

Ayvazo, S. \& Ward, P. (2011). Pedagogical Content Knowledge of Experienced Teachers in Physical Education: Functional Analysis of Adaptations. Research Quarterly for Exercise and Sport, vol. 82, n. 4, 675-684. 
Estudios Pedagógicos XL, $\mathrm{N}^{\circ}$ 2: 65-82, 2014

SIGNIFICADOS OTORGADOS A LA EVALUACIÓN DE LA ENSEÑANZA Y EL APRENDIZAJE. INTERPRETACIÓN A PARTIR DE UN GRUPO DE MAESTROS DE EDUCACIÓN FÍSICA

Azevedo, E. (2009). Percepções dos professores de educação física sobre as orientações curriculares: Estudo de caso dos colégios de aplicação no sul do Brasil. Tesis Doctoral, Universidade do Minho, Braga, Portugal.

Brown, G. (2002). Teachers' Conceptions of Assessment. Tesis Doctoral, University of Auckland, Auckland, New Zealand.

Cabezas, D., González, C. y Carpintero, E. (2009). Evaluar la evaluación: Cuestionario sobre prácticas de evaluación. Revista de Psicología y Educación. eduPsykhé, vol. 8, n. 1, 51-61.

Carreiro da Costa, F. (2008). Issues in Research on Teaching in Physical Education. In J. Seghers \& H. Vangrunderbeek (Eds.), Physical Education Research. What is the Evidence? (pp. 11-25). Leuven: Acco Leuven/ Voorburg.

(2004). El pensamiento del profesorado en el proceso enseñanza-aprendizaje en educación física. Revista Educación Física y Deporte, vol. 23, n. 2, 41-60.

Clark, C. \& Peterson, P. (1990). Procesos de pensamiento de los docentes. En M. Wittrock (Ed.), La investigación de la enseñanza III (pp. 442-539). Barcelona: Paidós.

Correa, M. y Martínez, N. (2006). El pensamiento del profesor de Educación Física: Un acercamiento a las prácticas educativas institucionales. Lúdica Pedagógica, vol. 2, n. 11, 34-51.

Denzin, N. \& Lincoln, Y. (2005). Introduction: The Discipline and Practice of Qualitative Research. In N. Denzin \& Y. Lincoln (Eds.), Handbook of Qualitative Research ( ${ }^{\text {rd }}$ Ed.) (pp. 1-32). Thousand Oaks, CA: Sage.

Desrosiers, P., Genet-Volet, Y. \& Godbout, P. (1997). Teachers Assessment Practices Viewed through the Instruments Used in Physical Education Classes. Journal of Teaching in Physical Education, vol. 16, n. 2, 211-228.

Díaz, J. (2005). La evaluación formativa como instrumento de aprendizaje en Educación Física. Barcelona: Inde.

(2001). El proceso de toma de decisiones en la programación de la educación física en las etapas obligatorias de educación. Una aportación a la formación del profesorado. Tesis Doctoral, Universitat Autònoma de Barcelona, Barcelona, España.

Ennis, C. \& Zhu, W. (1991). Value Orientations: A Description of Teachers' Goals for Student Learning. Research Quarterly for Exercise and Sport, vol. 62, n. 1, 33-40.

Fernández, J. (2007). Estudio del pensamiento del profesor de Educación Física en las etapas de primaria y secundaria. Tesis Doctoral, Universidad Complutense de Madrid, Madrid, España.

García, H. (2003). Antecedentes, evolución y aportaciones de la investigación del pensamiento y conocimiento del profesor de educación física. Adaxe: Revista de Estudos e Experiencias Educativas, n. $19,105-133$

Gimeno, J. (1992). La evaluación en la enseñanza. En J. Gimeno y A. Pérez (Eds.), Comprender y transformar la enseñanza (pp. 334-397). Madrid: Morata.

Goetz, J. y LeCompte, M. (1988). Etnografía y diseño cualitativo en investigación educativa. Madrid: Morata.

Guío, F. (2012). Educación Física: Tendencias y prácticas en evaluación de los aprendizajes en colegios distritales de Usaquén. Revista Educación Física y Deporte, vol. 31, n. 1, 863-870.

Habermas, J. (1987). Teoría de la acción comunicativa. Racionalidad de la acción y racionalización social (Vol. I). Madrid: Taurus.

(1982). Conocimiento e interés. Madrid: Taurus.

Hein, E., Vieira, J. y Mendes, J. (2007). Metamorfoses na avaliação em Educação Física: Da formação inicial à prática pedagógica escolar. Movimento, vol. 13, n. 2, 55-76.

Henrique, J. (2004). Processos mediadores do professor e do aluno: Uma abordagem qualiquantitativa do pensamento do professor, da interacção pedagógica e das percepções pessoais do aluno na disciplina de educação física. Tesis Doctoral, Universidade Técnica de Lisboa, Lisboa, Portugal.

Hernández, J. y López, A. (2004). Evaluación de la enseñanza: Análisis y propuestas. En J. Hernández, R. Velásquez, D. Alonso, F. Castejón, I. Garoz, C. López, A. López, A. Maldonado y M. 
Martínez (Eds.), La evaluación en educación física. Investigación y práctica en el ámbito escolar (pp. 49-76). Barcelona: Graó.

Jackson, P. (1992). La vida en las aulas (2 $2^{\mathrm{a}}$ Ed.). Madrid: Morata.

Januário, C. (1992). O pensamento do professor. Relação entre as decisões pré-interactivas, os comportamentos interactivos de ensino em educação física. Tesis doctoral, Universidade Técnica de Lisboa, Lisboa, Portugal.

Jinhong, J. (2012). The Focus, Role, and Meaning of Experienced Teachers' Reflection in Physical Education. Physical Education and Sport Pedagogy, vol. 17, n. 2, 157-175.

López-Pastor, V., Kirk, D., Lorente-Catalán, E., MacPhail, A. \& Macdonald, D. (2013). Alternative Assessment in Physical Education: A Review of International Literature. Sport, Education and Society, vol. 18, n. 1, 57-76.

López, V. (2006). La evaluación en Educación Física. Revisión de los modelos tradicionales y planteamiento de una alternativa: La evaluación formativa compartida. Madrid: Miño y Dávila. . (2004). La participación del alumnado en los procesos evaluativos: La autoevaluación y la evaluación compartida en Educación Física. En A. Fraile (Ed.), Didáctica de la Educación Física. Una perspectiva crítica y transversal (pp. 265-290). Madrid: Biblioteca Nueva. (2000). Buscando una evaluación formativa en educación física: Análisis crítico de la realidad existente, presentación de una propuesta y análisis general de su puesta en práctica. Apunts. Educació Física i Esports, vol. 64, n. 4, 16-26.

(1999). Prácticas de evaluación en educación física: Estudio de casos en primaria, secundaria y formación del profesorado. Valladolid: Universidad de Valladolid.

MacPhail, A. \& Halbert, J. (2010). 'We had to do Intelligent Thinking during Recent PE': Students' and Teachers' Experiences of Assessment for Learning in Post-primary Physical Education. Assessment in Education: Principles, Policy \& Practice, vol. 17, n. 1, 23-39.

Marcelo, C. (1987). El pensamiento del profesor. Barcelona: Ceac.

Myers, M. \& Newman, M. (2007). The Qualitative Interview in IS Research: Examining the Craft. Information and Organization, vol. 17, n. 1, 2-26.

Ní Chóinín, D. \& Cosgrave, C. (2012). Implementing Formative Assessment in Primary Physical Education: Teacher Perspectives and Experiences. Physical Education and Sport Pedagogy, vol. 18, n. 2, 219-233.

Penney, D., Brooker, R., Hay, P. \& Gillespie, L. (2009). Curriculum, Pedagogy and Assessment: Three Message Systems of Schooling and Dimensions of Quality Physical Education. Sport, Education and Society, vol. 14, n. 4, 421-442.

Pérez, A., Heras, C. y Herrán, I. (2008). Evaluación formativa en la educación secundaria obligatoria. Su aplicación a una unidad didáctica de deportes colectivos en el marco del estilo actitudinal. Revista Española de Educación Física y Deportes, vol. 9, n. julio - diciembre, 45-66.

Pradas, R. (2010). El estudio del pensamiento del profesorado sobre la toma de decisiones interactivas: Análisis de un caso en educación física escolar. Cultura y Educación, vol. 22, n. 1, 21-36.

Salinas, D. (2002). ¡Mañana examen! La evaluación: Entre la teoría y la realidad. Barcelona: Graó.

Sandoval, C. (1996). Investigación cualitativa. Bogotá: ICFES.

Santos, M. (2003). Una flecha en la diana. La evaluación como aprendizaje. Madrid: Narcea. Aljibe. . (1995). La evaluación: Un proceso de diálogo, comprensión y mejora (2ª Ed.). Málaga:

Schön, D. (1992). La formación de profesionales reflexivos. Hacia un nuevo diseño de la enseñanza y el aprendizaje en los profesores. Barcelona: Paidós.

Shulman, L. (1989). Paradigmas y programas de investigación en el estudio de la enseñanza. Una perspectiva contemporánea. En M. Wittrock (Ed.), La investigación de la enseñanza I (pp. 9-91). Barcelona: Paidós.

Strauss, A. y Corbin, J. (2002). Bases de la investigación cualitativa: Técnicas y procedimientos 
para desarrollar la teoría fundamentada. Medellín: Facultad de Enfermería de la Universidad de Antioquia.

Taylor, S. \& Bogdan, R. (1987). Introducción a los métodos cualitativos de investigación. Barcelona: Paidós.

Tristán, J. (2010). La relación de la planificación en la interacción sobre los comportamientos del profesorado principiante y con experiencia así como la de sus alumnos en un estudio de casos. Tesis doctoral, Universidad de Murcia, Murcia, España.

Ureña, N., Ureña, F., Velandrino, A. y Alarcón, F. (2006). Resultados de un caso práctico de autoevaluación en educación física en la etapa de primaria Apunts. Educació Física i Esports, vol. 86, n. 4, 22-33.

Uribe, I., Gallo, L. y Castro, J. (2004). La motricidad como potenciadora de las metas del desarrollo. En I. Uribe, L. Gallo, J. Castro, A. Correa, P. Gallego y F. Vélez (Eds.), Sentidos de la motricidad en la promoción de la salud. Percepciones desde las familias en los municipios de Alejandría y Guatapé (Antioquia) (pp. 207-261). Medellín: Kinesis.

Velázquez, R. y Hernández, J. (2004). Evaluación en educación y evaluación del aprendizaje en educación física. En J. Hernández y R. Velázquez (Eds.), La evaluación en educación física. Investigación y práctica en el ámbito escolar (pp. 11-47). Barcelona: Graó.

Vera, J. (2010). Dilemas en la negociación del currículum con el alumnado a partir de la cesión de responsabilidad de la evaluación en el aula de Educación Física. Revista de Investigación en Educación, n. 7, 72-82.

Vera, J. y Moreno, J. (2007). El pensamiento del profesorado en educación física ante la cesión de responsabilidad en la evaluación del alumnado. Profesorado. Revista de Currículum y Formación del Profesorado, vol. 11, n. 2, 1-15.

Woods, P. (1987). La escuela por dentro. Etnografía en la investigación cualitativa. Madrid: Paidós/MEC. 